\title{
Qual o prognóstico da artrodese tríplice quando utilizada no tratamento do pé plano adquirido do adulto (PPAA)?*
}

\section{What is the Prognosis of Triple Arthrodesis in the Treatment of Adult Acquired Flatfoot Deformity (AAFD)?}

\author{
Daiana Kerry Picanço Gobbo ${ }^{10}$ Nilson Roberto Severino ${ }^{1}$ Ricardo Cardenuto Ferreira ${ }^{1}$ \\ ${ }^{1}$ Departamento de Ortopedia e Traumatologia, Santa Casa de São \\ Paulo, São Paulo, SP, Brasil \\ Rev Bras Ortop 2019;54:275-281. \\ Address for correspondence Daiana Kerry Picanço Gobbo, MD, \\ Departamento de Ortopedia e Traumatologia, Santa Casa de São \\ Paulo, Rua Barata Ribeiro, 380 - Cj 64 - sexto andar - Bela Vista, São \\ Paulo, SP, 01308-000, Brasil (e-mail: daianagobbo@yahoo.com.br).
}

\section{Resumo}

Palavras-chave

- pé chato/etiologia

- pé chato/cirurgia

- artrodese

- deformidades adquiridas do pé
Objetivo Avaliar a capacidade da artrodese tríplice de aliviar as principais queixas dos pacientes que apresentam pé plano adquirido do adulto (PPAA): 1) dor incapacitante localizada no médio e retropé; 2) deformidades marcadas pelo colapso do arco medial, valgo, abdução e supinação.

Método Avaliamos 17 pacientes (20 pés) portadores de PPAA em estado avançado que foram submetidos à correção cirúrgica pela artrodese tríplice modelante. A média de idade dos pacientes no momento da cirurgia foi de 62 anos (variação de 38 a 79 anos), e o tempo médio de seguimento foi de 43 meses (variação de 18 a 84 meses). Utilizamos critérios clínicos empregando a escala visual analógica da dor (EVAD) e a escala funcional da American Orthopaedic Foot and Ankle Society (AOFAS, na sigla em inglês) do retropé para avaliar a eficácia da cirurgia.

Resultados A dor residual mensurada pela EVAD foi de três pontos, em média. Observamos incremento médio de $23 \%$ nos valores da escala AOFAS do retropé após o tratamento cirúrgico. A correção das deformidades foi satisfatória em 10 de 20 pés; parcialmente satisfatória em 4 de 20 pés; parcialmente insatisfatória em 5 de 20 pés; e insatisfatória em 1 de 20 pés.

Conclusão Apesar da artrodese tríplice modelante indicada no tratamento do PPAA em estágio avançado apresentar alto índice de consolidação óssea, a correção incompleta das deformidades pré-existentes e a persistência de dor residual contribuíram para a elevada taxa de decepção dos pacientes com o resultado da cirurgia.

\footnotetext{
Trabalho desenvolvido no Grupo de Cirurgia do Pé e Tornozelo do Departamento de Ortopedia e Traumatologia da Santa Casa de São Paulo, São Paulo, SP, Brasil.

(DD) Daiana Kerry Picanço Gobbo's ORCID is https://orcid.org/0000-00017552-6590.
}

received

January 1, 2019

accepted

March 29, 2019
DOI https://doi.org/

10.1055/s-0039-1692445. ISSN 0102-3616.
Copyright $\odot 2019$ by Sociedade Brasileira License terms de Ortopedia e Traumatologia. Published by Thieme Revnter Publicações Ltda, Rio de Janeiro, Brazil 


\begin{abstract}
Objective The purpose of the present study is to evaluate the ability of triple arthrodesis to eliminate the main complaints presented by patients with adult acquired flatfoot deformity (AAFD): 1) disabling pain in the hindfoot; 2) major deformities such medial arch collapse, valgus, abduction and supination.

Methods A total of 17 patients (20 feet) with advanced stage of AAFD who underwent surgical correction by triple arthrodesis were evaluated after a mean follow-up of 43 (range 18-84) months. The average age of the patients at the time of the surgery was of 62 years old (range 38-79 years old). The visual analogue pain scale (VAS) and the American Orthopaedic Foot and Ankle Society (AOFAS) hindfoot score were used to assess the final results.

Results According to the VAS, the average residual pain score was three; The average increase in the AOFAS hindfoot score points was of $23 \%$ after the surgery; and the correction of deformities was satisfactory in 10 out of 20 feet; partially satisfactory in 4

\section{Keywords}

- flatfoot/etiology

- flatfoot/surgery

- arthrodesis

- acquired foot deformities

out of 20 feet; partially unsatisfactory in 5 out of 20 feet; and unsatisfactory in 1 out of 20 feet.

Conclusion Despite the high index of bone fusion after triple arthrodesis, witch is the gold standard treatment in advanced stages of AAFD, the incomplete correction of major deformities and the persistence of residual pain contributed to a high index of disappointment of the patients with the surgical results.
\end{abstract}

\section{Introdução}

A artrodese tríplice modelante é o tratamento cirúrgico padrão indicado nos estágios avançados do pé plano adquirido do adulto (PPAA), como no caso das lesões classificadas como estágio III. Nesta situação, a rigidez articular está frequentemente associada à artrose difusa do retropé e é acompanhada de deformidades típicas, destacando-se: 1) colapso do arco medial; 2) valgo do retropé; 3) abdução e supinação do antepé. ${ }^{1-8} \mathrm{~A}$ artrodese tríplice também pode ter sua indicação estendida para pacientes mais idosos ou com sobrepeso, mesmo quando o estágio evolutivo do PPAA encontra-se ainda numa fase intermediária (estágio II), na qual normalmente poderiam ser indicadas cirurgias que preservassem algum grau de movimento articular. ${ }^{8-13}$ Nos pacientes obesos, as cirurgias corretivas que envolvem osteotomias associadas a transferências musculotendíneas apresentam maior chance de falha com recidivas. ${ }^{8,12}$ Da mesma maneira, pacientes com idade avançada e baixa demanda funcional também podem ser bons candidatos à artrodese tríplice, uma vez que esta cirurgia contribuiria, em tese, para aliviar os sintomas dolorosos com reduzido risco de recidiva das deformidades, evitando assim a possibilidade de uma segunda operação. ${ }^{8-16}$

O objetivo do presente estudo é avaliar a capacidade da artrodese tríplice modelante de aliviar os sintomas dolorosos e as deformidades relacionados ao PPAA. Nossa hipótese é a de que esta cirurgia é capaz de propiciar melhora substancial na intensidade da dor e no alinhamento das extremidades deformadas.

\section{Casuística e Método}

No período compreendido entre janeiro e março de 2015, convocamos todos os pacientes cadastrados no banco de dados do nosso hospital cujo diagnóstico era PPAA e que haviam sido submetidos a tratamento cirúrgico com artrodese tríplice modelante. Excluímos pacientes com diagnóstico de doenças reumatológicas associadas e aqueles cujo tempo mínimo de seguimento pós-operatório era $<12$ meses. Responderam à nossa convocação 17 pacientes (15 femininos e 2 masculinos). Em 3 pacientes, a cirurgia foi realizada bilateralmente, totalizando 20 pés operados. No momento da cirurgia, a média de idade dos pacientes foi de 62 anos (variando de 38 a 79 anos) e o IMC médio foi 31 (variando de 23 a 42). Todos pacientes foram operados de maneira convencional sob raquianestesia e utilizando garrote pneumático na raiz da coxa com pressão de $300 \mathrm{~mm}$ Hg. Empregamos dupla via de acesso cirúrgico, uma lateral e outra medial. Após dissecção por planos, as articulações subtalar, talonavicular e calcaneocubóide foram identificadas e, em seguida, cunhas ósseas de tamanhos apropriados foram removidas das superfícies articulares para permitir uma adequada coaptação e, ao mesmo tempo, corrigir as deformidades essenciais presentes no PPAA. Fixação interna com parafusos foi empregada em todos os casos. Imobilização com bota gessada foi mantida durante 12 semanas. Carga sobre o membro operado foi permitida a partir da $7^{a}$ semana. Após a remoção do gesso, indicamos imobilização com bota removível por 4 semanas adicionais, período em que os pacientes iniciaram fisioterapia assistida.

Durante o transcorrer do seguimento pós-operatório, identificamos a ocorrência de complicações menores em cinco pés, destacando-se: deiscência da ferida operatória e necrose cutânea parcial nas bordas da incisão cirúrgica. $O$ tratamento destas lesões consistiu no desbridamento local associado à prescrição de antibioticoterapia sistêmica oral, 
resultando em cicatrização completa sem maiores complicações.

Para avaliação do resultado clínico-funcional do tratamento, utilizamos a escala da American Orthopaedic Foot and Ankle Society (AOFAS, na sigla em inglês) corrigida para o retropé $^{17,18}$ (variação pré-operatória de 0 a 100; variação pós-operatória de 0 a 94); a escala visual analógica para dor $(E V A D)^{19}$; e o grau de satisfação dos pacientes com o resultado do tratamento (completamente satisfeito; satisfeito com mínimas restrições; satisfeito com maiores restrições; e insatisfeito).

Para a avaliação da capacidade da cirurgia de corrigir as principais deformidades clínicas presentes no PPA, um examinador independente mensurou e comparou as imagens fotográficas pré-operatórias dos pés dos pacientes com os achados clínicos da avaliação pós-operatória. A variação no grau do colapso do arco, do valgo do retropé, e da abdução e supinação do médio e antepé foi cuidadosamente analisada, e seu grau de correção foi classificado pelo examinador como: 1) completamente satisfatório, quando as quatro deformidades mencionadas foram adequadamente corrigidas segundo os parâmetros clínicos de normalidade descritos; 2) parcialmente satisfatório, quando pelo menos três das quatro deformidades foram consideradas adequadamente corrigidas; 3) parcialmente insatisfatório, quando apenas duas das quatro deformidades foram consideradas adequadamente corrigidas; e 4) completamente insatisfatório, quando apenas uma ou nenhuma deformidade das quatro deformidades foram corrigidas (-Fig. 1).

Além dos critérios clínicos, utilizamos também critérios radiográficos para avaliar a capacidade da artrodese tríplice modelante de obter a correção das principais deformidades associadas ao PPAA. ${ }^{20,21}$ Nas imagens radiográficas simples realizadas com apoio nas incidências dorsoplantar, lateral e axial perna-pé, mensuramos a diferença pré- e pós-operatória dos seguintes parâmetros: 1) altura do arco plantar longitudinal medial; 2) inclinação em valgo do retropé; 3 ) inclinação em abdução antepé (-Fig. 2).

O trabalho foi aprovado pela nossa instituição sob o número CAAE 43134015.0.0000.5479.

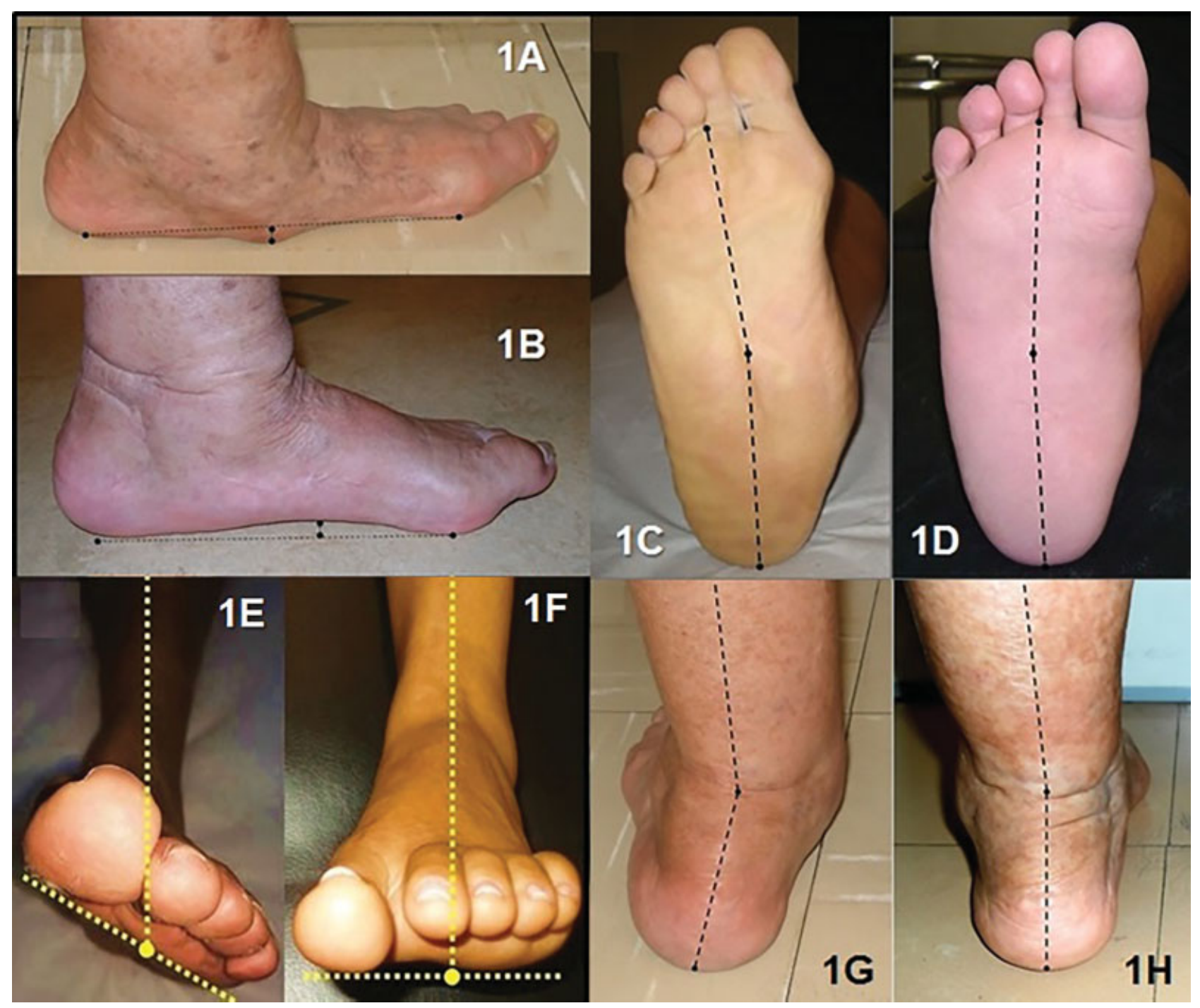

Fig. 1 Vista medial do pé esquerdo mostrando o colapso do arco plantar medial (1A) que foi corrigido após a cirurgia (1B). Vista plantar do pé direito mostrando a abdução (1C) corrigida após a cirurgia (1D). Vista frontal do pé direito mostrando grave deformidade em supinação (1E) corrigida após a cirurgia (1F). Vista posterior do retropé mostrando acentuada deformidade em valgo (1G) corrigida após a cirurgia (1H). 


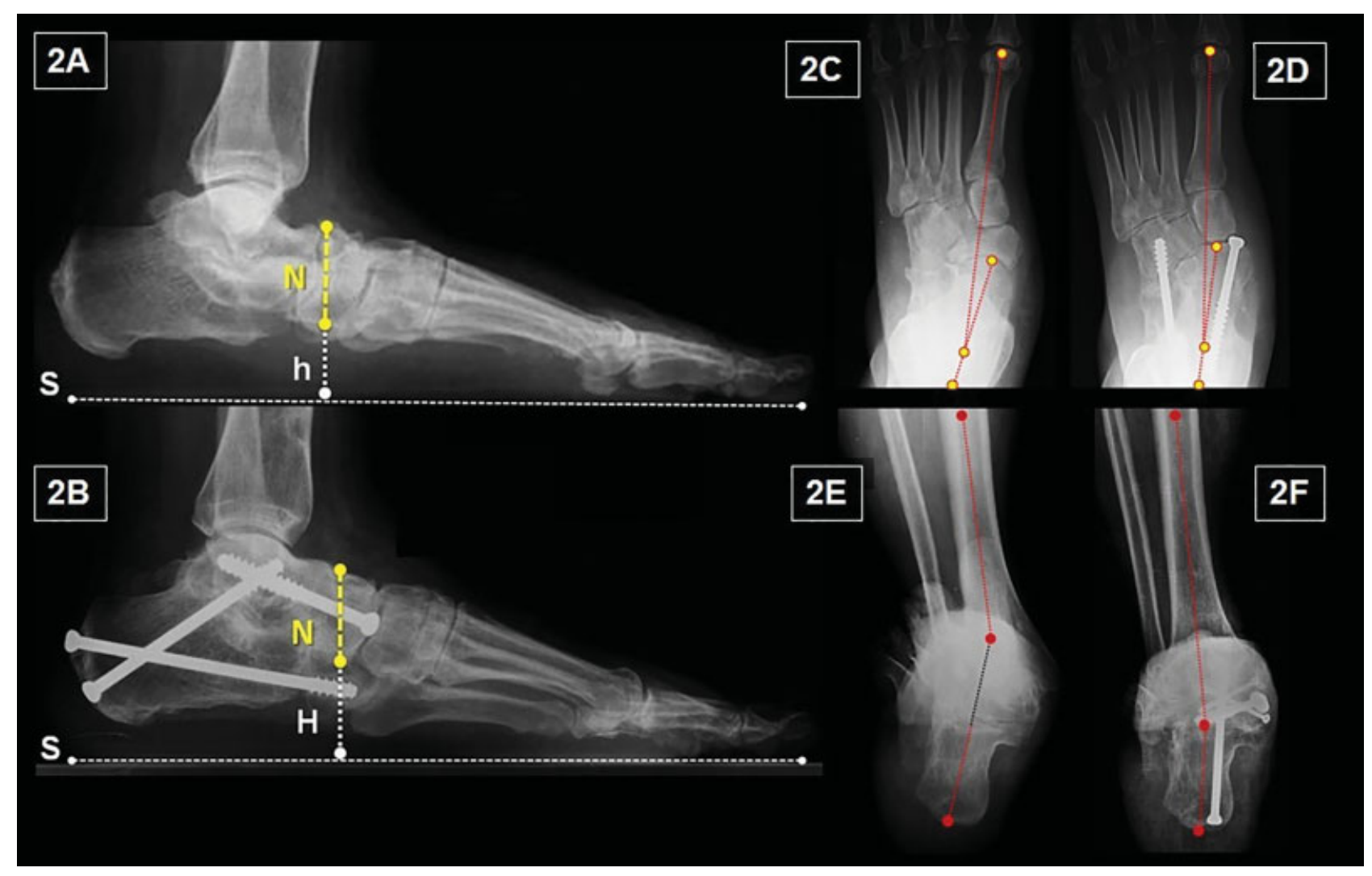

Fig. 2 Imagem radiográfica lateral do pé e do tornozelo mostrando colapso do arco medial (2A) corrigido após a cirurgia (2B). A altura do arco tanto no pré- (h) quanto no pós-operatório (H) é determinada pela distância da margem inferior do eixo do navicular (N) em direção ao solo (S). Imagem radiográfica anteroposterior do pé mostrando a abdução antes (2C) e após a correção cirúrgica (2D). Imagem radiográfica axial perna-pé mostrando a deformidade em valgo (2E) corrigida com a cirurgia (2F).

\section{Resultados}

Consolidação óssea completa, marcada pela presença de trabeculado ósseo cruzando o local da artrodese nas imagens radiográficas, ocorreu em 19 dos 20 pés operados (95\%). Apesar do elevado índice de consolidação, a cirurgia não garantiu a eliminação completa da dor. Os resultados obtidos segundo a avaliação da EVAD identificou presença de dor residual numa intensidade de 3 de um máximo de 10 pontos possíveis (variação de 0 a 6 pontos). Com relação ao resultado clínico-funcional mensurado pela escala AOFAS do retropé, houve um incremento médio de 18 pontos após a cirurgia. A pontuação média pré-operatória aumentou de 56/100 (variação de 43 a 69) para 74/94 (variação de 55 a 90). Este incremento proporcional na pontuação correspondeu à melhora proporcional de 23\% nos parâmetros mensurados pela escala AOFAS da condição pré-operatória para a condição pós-operatória. Considerando a satisfação dos pacientes com o resultado da cirurgia, identificamos que satisfação completa foi relatada em 7 pés (35\%); satisfação com mínimas restrições em 5 pés (25\%); e satisfação com maiores restrições em 8 pés (40\%). Nenhum paciente considerou que o resultado final do tratamento tenha sido insatisfatório (-Tabela $\mathbf{1}$ ).

Segundo a avaliação clínica do examinador considerando a capacidade da cirurgia de corrigir as principais deformidades presentes no PPAA, os resultados obtidos foram: completamente satisfatórios em 10 de 20 pés (50\%); parcialmente satisfatórios em 4 de 20 pés (20\%); parcialmente insatisfatórios em 5 de 20 pés (25\%); e completamente insatisfatório em 1 de 20 pés (5\%) (-Tabela 1).

$\mathrm{Na}$ avaliação radiográfica da capacidade da cirurgia de corrigir as principais deformidades presentes no PPAA, os resultados obtidos foram: 1) o aumento percentual médio proporcional na altura do arco plantar longitudinal medial após a cirurgia foi de 34\%; 2) a diminuição percentual média da inclinação em valgo do retropé foi de $27 \%$ (a angulação média no pré-operatório passou de $11^{\circ}$ para $8^{\circ}$ no pósoperatório); 3) diminuição percentual média da inclinação em abdução antepé foi de $80 \%$ (a angulação média no préoperatório passou de $15^{\circ}$ para $3^{\circ}$ no pós-operatório).

\section{Discussão}

A grande incidência do PPAA acomete pacientes na $4^{\underline{a}}$ e $5^{\underline{a}}$ décadas da vida, com sobrepeso e pouco ativos, sendo predominante no sexo feminino. ${ }^{22-27}$ Neste grupo de pacientes, a queixa principal costuma ser dor incapacitante, enquanto as deformidades que acompanham o PPAA aparecem como queixa secundária relacionadas, principalmente, à dificuldade em acomodar o pé dentro do calçado convencional, além do excessivo desgaste do próprio calçado associado ao apoio na região medial do solado. 0 princípio do tratamento cirúrgico considera que a melhora dos sintomas 
Tabela 1 Distribuição dos 17 pacientes (20 pés) segundo aspectos epidemiológicos, complicações pós-operatórias, tempo de seguimento, e resultados clínico-funcionais

\begin{tabular}{|c|c|c|c|c|c|c|c|c|c|}
\hline Caso & $\begin{array}{l}\text { Gênero, } \\
\text { idade, lado }\end{array}$ & $\begin{array}{l}\text { Classificação } \\
\text { GRAU II ou III }\end{array}$ & IMC & $\begin{array}{l}\text { Complicações } \\
\text { Imediatas / } \\
\text { Tardias }\end{array}$ & $\begin{array}{l}\text { Tempo de } \\
\text { Seguimento }\end{array}$ & $\begin{array}{l}\text { AOFAS } \\
\text { PRÉ / PÓS }\end{array}$ & EVAD & $\begin{array}{l}\text { Satisfação } \\
\text { Pessoal }\end{array}$ & $\begin{array}{l}\text { Correção das } \\
\text { deformidades }\end{array}$ \\
\hline 1 & $\mathrm{~F}, 52, \mathrm{D} / \mathrm{E}$ & II & $31,1 / 24,8$ & não & 48 e 18 meses & 67 para 72 e 73 & 5 e 5 & Menor restrição & $\begin{array}{l}\text { Satisfatorio parcial// } \\
\text { Satisfatorio total }\end{array}$ \\
\hline 2 & F, 54, E & II & 39,6 & não & 24 meses & 52 para73 & 4 & Satisfeito & Satisfatório total \\
\hline 3 & $\mathrm{~F}, 75, \mathrm{E}$ & 11 & 27,3 & não & 30 meses & 57 para 80 & 3 & Satisfeito & Satisfatório total \\
\hline 4 & $F, 47, E$ & $\|$ & 31,8 & não & 47 meses & 55 para 81 & 2 & Maior restrição & Satisfatório total \\
\hline 5 & $\mathrm{~F}, 68, \mathrm{E}$ & II & 27,5 & não & 49 meses & 69 para 80 & 0 & Satisfeito & Satisfatório total \\
\hline 6 & $\mathrm{~F}, 63, \mathrm{D}$ & II & 22,9 & não & 84 meses & 62 para 83 & 3 & Satisfeito & Insatisfatório parcial \\
\hline 7 & $F, 49, E$ & III & 41,9 & não & 12 meses & 56 para 75 & 2 & Satisfeito & Satisfatório total \\
\hline 8 & F, 75, D & III & 24,2 & DFO & 12 meses & 43 para 70 & 2 & Satisfeito & Insatisfatório parcial \\
\hline 9 & $F, 69, E$ & III & 42,0 & não & 24 meses & 58 para 78 & 6 & Maior restrição & Satisfatório total \\
\hline 10 & $\mathrm{~F}, 70, \mathrm{D} / \mathrm{E}$ & III & $38,7 / 38,7$ & não & 24 e 48 meses & $\begin{array}{l}55 \text { para } 85 \\
\text { e } 52 \text { para } 90\end{array}$ & 4 e 4 & Menor restrição & $\begin{array}{l}\text { Satisfatório total/ } \\
\text { Satisfatório parcial }\end{array}$ \\
\hline 11 & $\mathrm{M}, 79, \mathrm{D} / \mathrm{E}$ & III & $25,0 / 25,0$ & não & 26 e 72 meses & $\begin{array}{l}58 \text { para } 81 \\
\text { e } 51 \text { para } 80\end{array}$ & 3 e 3 & Maior restrição & $\begin{array}{l}\text { Satisfatório total/ } \\
\text { Satisfatório parcial }\end{array}$ \\
\hline 12 & $\mathrm{~F}, 45, \mathrm{E}$ & III & 35,2 & DFO & 35 meses & 57 para 65 & 0 & Maior restrição & Satisfatório total \\
\hline 13 & $\mathrm{~F}, 70, \mathrm{E}$ & III & 33,2 & não & 43 meses & 44 para 55 & 3 & Maior restrição & Insatisfatório parcial \\
\hline 14 & F, 70, D & III & 30,1 & $\begin{array}{l}\text { DFO e } \\
\text { pseudartrose }\end{array}$ & 48 meses & 49 para 55 & 3 & Menor restrição & Insatisfatório \\
\hline 15 & $F, 61, E$ & III & 31,0 & não & 60 meses & 46 para 76 & 2 & Satisfeito & Insatisfatório parcial \\
\hline 16 & $F, 58, D$ & III & 26,5 & DFO & 75 meses & 66 para 73 & 3 & Maior restrição & Insatisfatório parcial \\
\hline 17 & $\mathrm{M}, 38, \mathrm{E}$ & III & 24,0 & não & 90 meses & 54 para 62 & 5 & Maior restrição & Satisfatório parcial \\
\hline
\end{tabular}

Abreviações: AOFAS, escala numérica para avaliação clínico-funcional do retropé da American Orthopedic Foot and Ankle Society; D, direito; DFO, deiscência da ferida operatória; E, esquerdo; EVAD, escala visual analógica da dor; F, feminino; M, masculino.

clínicos depende da correção das deformidades e do restabelecimento da força inversora do pé. Nos anos mais recentes, uma série de publicações envolvendo estudos com séries de casos clínicos (nível de evidência científica IV) tem enfatizado a importância de substituir o tendão tibial posterior degenerado e corrigir as deformidades típicas do PPAA por meio de múltiplas osteotomias corretivas, desde que ainda exista mobilidade remanescente suficiente para permitir o alinhamento adequado do pé, como ocorre nas lesões no estágio II. ${ }^{22-26}$ Esta tendência tem como raciocínio básico poupar as articulações e preservar a mobilidade do retropé, além de evitar complicações tardias da artrodese tríplice, principalmente à artrose secundária das articulações adjacentes, em especial o tornozelo e as demais articulações remanescentes no médio-tarso. ${ }^{22,23}$ A substituição do tendão tibial posterior degenerado e insuficiente pelo tendão flexor longo dos dedos é a opção preferencial. ${ }^{22}$ Infelizmente, a recorrência das deformidades do PPAA após a realização de múltiplas osteotomias do tarso e a substituição do tendão tibial posterior pelo flexor longo dos dedos não é um acontecimento raro. Isto é particularmente relatado nos pacientes portadores de doenças reumatológicas, com sobrepeso ou idade avançada, sendo necessário considerar a artrodese tríplice como alternativa de tratamento cirúrgico quando estes fatores de risco estiverem presentes. ${ }^{22}$

A artrodese tríplice modelante constitui o tratamento cirúrgico padrão nos pacientes que não respondem ao tratamento conservador do PPAA e já se encontram em uma fase mais avançada da doença, mais especificamente no estágio III. Eventualmente, sua indicação pode ser estendida também para um grupo específico de pacientes que ainda não desenvolveram deformidades rígidas acompanhadas de artrose (estágio II), mas que têm como particularidade a idade avançada ( $>65$ anos) ou a obesidade (IMC > 30)..$^{8,12}$ Apesar de numerosos estudos sugerirem que a obesidade possa gerar impacto negativo sobre os resultados cirúrgicos em diversas articulações do corpo, ${ }^{28-31}$ existem poucos estudos sobre o seu efeito nas cirurgias ortopédicas do pé e do tornozelo. Recentemente, Soukup et $\mathrm{al}^{32}$ não identificaram diferenças significantes no resultado, considerando pacientes com peso normal, sobrepeso e obesos. Segundo estes autores, o tratamento do PPA grau II empregando a transferência tendínea associada a múltiplas osteotomias poderia também ser indicada para pacientes com IMC $>25$. Entretanto, os resultados deste estudo devem ser analisados com cautela devido ao tempo médio de seguimento pós-operatório ter sido relativamente curto, de apenas 3 anos.

Com relação ao resultado do tratamento cirúrgico do PPAA, vale a pena ressaltar que nem sempre todos os sintomas dolorosos são completamente eliminados pela cirurgia, tanto as que corrigem as deformidades empregando osteotomias tarsais múltiplas quanto aquelas nas quais a correção é acompanhada da estabilização tarsal definitiva pela artrodese tríplice. ${ }^{12}$ A persistência da dor residual é causa comum de certo grau de insatisfação dos pacientes com o resultado do tratamento cirúrgico, 
independentemente de terem sido realizadas as osteotomias ou artrodeses. ${ }^{33,34}$ Vale a pena ressaltar que, na nossa série de casos, a consolidação óssea foi obtida em $95 \%$ dos pés operados mas, apesar disto, a dor residual permaneceu em um patamar médio de intensidade de $\sim 3$ pontos na EVAD. Este achado reforça a necessidade de informar previamente ao paciente que a cirurgia não necessariamente propiciará a eliminação completa do desconforto doloroso, mesmo que a consolidação óssea da artrodese seja completa. A persistência da dor residual pode ter refletido diretamente no grau de satisfação pessoal dos pacientes que compuseram nossa série de casos com relação ao resultado final da cirurgia. Dos 20 pés operados, a satisfação completa foi relatada em apenas 7 (35\%), enquanto satisfação com menores ou maiores restrições foi relatada em 13 (65\%).

Uma possível explicação para a persistência da dor pode ser atribuída ao fato da cirurgia não ter sido capaz de corrigir adequadamente as deformidades pré-existentes. Considerando que, na avaliação clínica pós-operatória, apenas metade dos pés foram considerados completamente corrigidos, a presença de deformidades residuais poderia ser responsabilizada pela manutenção da dor. Segundo dados da avaliação radiográfica, o incremento percentual médio na altura do arco plantar após a cirurgia restringiu-se apenas a $\sim 34 \%$; além disso, o valgo residual persistiu em um patamar acima da média, girando em $\sim 8^{\circ}$, apesar de o tratamento cirúrgico ter proporcionado um índice médio de correção de $27 \%$. A única deformidade que foi substancialmente corrigida na nossa série de casos foi a abdução, que praticamente atingiu o valor médio dentro da faixa aceita como adequada, ou seja, $\sim 3^{\circ}$.

A natureza retrospectiva do presente estudo e o pequeno tamanho do grupo amostral conferem o ponto fraco do presente estudo. Entretanto, acreditamos que as informações obtidas podem ser úteis para considerar as limitações da artrodese tríplice modelante como forma de aliviar completamente os sintomas relacionados ao PPAA em estado de gravidade avançada. É preciso considerar que este tipo de cirurgia corretiva não é simples, e sua execução necessita adequada expertise do cirurgião em alcançar o posicionamento adequado do pé. A correção incompleta das graves deformidades após o tratamento cirúrgico pode ser um fator importante na manutenção da dor residual, mas sua real importância ainda não é completamente conhecida e não pode ser identificada no presente estudo.

\section{Conclusão}

Apesar da artrodese tríplice modelante indicada no tratamento do PPAA em estágio avançado apresentar alto índice de consolidação óssea, a correção incompleta das deformidades pré-existentes e a persistência de dor residual contribuíram para a elevada taxa de decepção dos pacientes com o resultado da cirurgia.

Conflitos de Interesses

Os autores declaram não haver conflitos de interesses.

\section{Referências}

1 Kulowski J. Tendovaginitis (tenosynovitis), general discussion and report of one case involving the posterior tibial tendon. J Miss State Med Assoc 1936;33:135-137

2 Lipscomb P. Non-suppurative tenosynovitis and paratendinitis. Instr Course Lect 1950;7:254

3 Taylor R, Sammarco VJ. Minimizing the role of fusion in the rigid flatfoot. Foot Ankle Clin 2012;17(02):337-349

4 Grunander TR, Thordarson DB. Results of calcaneocuboid distraction arthrodesis. Foot Ankle Surg 2012;18(01):15-18

5 Lin JS, Myerson MS. The management of complications following the treatment of flatfoot deformity. Instr Course Lect 2011; 60:321-334

6 Johnson KA, Strom DE. Tibialis posterior tendon dysfunction. Clin Orthop Relat Res 1989;(239):196-206

7 Myerson MS. Adult acquired flatfoot deformity: treatment of dysfunction of the posterior tibial tendon. J Bone Joint Surg Am 1996;78(05):780-792

8 Hatic SO II, Philbin TM. Management of the recurrent deformity in a flexible foot following failure of tendon transfer: is arthrodesis necessary? Foot Ankle Clin 2012;17(02):299-307

9 Gentchos CE, Anderson JG, Bohay DR. Management of the rigid arthritic flatfoot in the adults: alternatives to triple arthrodesis. Foot Ankle Clin 2012;17(02):323-335

10 Mosier SM, Pomeroy G, Manoli A II. Pathoanatomy and etiology of posterior tibial tendon dysfunction. Clin Orthop Relat Res 1999; (365):12-22

11 Schuh R, Salzberger F, Wanivenhaus AH, Funovics PT, Windhager $\mathrm{R}$, Trnka HJ. Kinematic changes in patients with double arthrodesis of the hindfoot for realignment of planovalgus deformity. J Orthop Res 2013;31(04):517-524

12 Ellington JK, Myerson MS. The use of arthrodesis to correct rigid flatfoot deformity. Instr Course Lect 2011;60:311-320

13 Bluman EM, Title CI, Myerson MS. Posterior tibial tendon rupture: a refined classification system. Foot Ankle Clin 2007;12(02): 233-249, v

14 Raikin SM, Winters BS, Daniel JN. The RAM classification: a novel, systematic approach to the adult-acquired flatfoot. Foot Ankle Clin 2012;17(02):169-181

15 Neville CG, Candidate MS, Houck JR. Science behind the use of orthotic devices to manage posterior tibial tendon dysfunction. Tech Foot Ankle Surg 2008;7(02):125-133

16 Ryerson E. Arthrodesing operations on the feet. J Bone Joint Surg Am 1923;5:453-471

17 Pinsker E, Daniels TR. AOFAS position statement regarding the future of the AOFAS Clinical Rating Systems. Foot Ankle Int 2011;32(09): $841-842$

18 Kitaoka HB, Alexander IJ, Adelaar RS, Nunley JA, Myerson MS, Sanders M. Clinical rating systems for the ankle-hindfoot, midfoot, hallux, and lesser toes. Foot Ankle Int 1994;15(07): 349-353

19 Gift AG. Visual analogue scales: measurement of subjective phenomena. Nurs Res 1989;38(05):286-288

20 Saltzman CL, el-Khoury GY. The hindfoot alignment view. Foot Ankle Int 1995;16(09):572-576

21 de Cesar Netto C, Schon LC, Thawait GK, da Fonseca LF, Chinanuvathana A, Zbijewski WB, et al. Flexible Adult Acquired Flatfoot Deformity: Comparison Between Weight-Bearing and Non-WeightBearing Measurements Using Cone-Beam Computed Tomography. J Bone Joint Surg Am 2017;99(18):e98

22 Flemister AS Jr, Baumhauer JF, Digiovanni BF. Flexor digitorum longus to posterior tibialis transfer with lateral column lengthening for stage ii posterior tibial tendon dysfunction. Tech Foot Ankle Surg 2007;6(01):22-29

23 Griend RV. Lateral column lengthening using a "Z" osteotomy of the calcaneus. Tech Foot Ankle Surg 2008;7(04):257-263 
24 Pomeroy GC, Manoli A II. A new operative approach for flatfoot secondary to posterior tibial tendon insufficiency: a preliminary report. Foot Ankle Int 1997;18(04):206-212

25 Myerson MS, Corrigan J. Treatment of posterior tibial tendon dysfunction with flexor digitorum longus tendon transfer and calcaneal osteotomy. Orthopedics 1996;19(05): 383-388

26 Hirose $\mathrm{CB}$, Johnson JE. Plantarflexion opening wedge medial cuneiform osteotomy for correction of fixed forefoot varus associated with flatfoot deformity. Foot Ankle Int 2004;25(08): 568-574

27 Holmes GB Jr, Mann RA. Possible epidemiological factors associated with rupture of the posterior tibial tendon. Foot Ankle 1992;13(02):70-79

28 Kerkhoffs GM, Servien E, Dunn W, Dahm D, Bramer JA, Haverkamp D. The influence of obesity on the complication rate and outcome of total knee arthroplasty: a meta-analysis and systematic literature review. J Bone Joint Surg Am 2012;94(20): 1839-1844
29 Linberg CJ, Sperling JW, Schleck CD, Cofield RH. Shoulder arthroplasty in morbidly obese patients. J Shoulder Elbow Surg 2009;18 (06):903-906

30 Dowsey MM, Liew D, Stoney JD, Choong PF. The impact of obesity on weight change and outcomes at 12 months in patients undergoing total hip arthroplasty. Med J Aust 2010;193(01):17-21

31 Chan CL, Villar RN. Obesity and quality of life after primary hip arthroplasty. J Bone Joint Surg Br 1996;78(01):78-81

32 Soukup DS, MacMahon A, Burket JC, Yu JM, Ellis SJ, Deland JT. Effect of Obesity on Clinical and Radiographic Outcomes Following Reconstruction of Stage II Adult Acquired Flatfoot Deformity. Foot Ankle Int 2016;37(03):245-254

33 Barrôco R, Nery C, Netto AA. Pé plano adquirido por disfunção do tibial posterior: resultados cirúrgicos. Rev Bras Ortop 2002;37 (06):211-218

34 Persaud S, Hentges MJ, Catanzariti AR. Occurrence of Lateral Ankle Ligament Disease With Stage 2 to 3 Adult-Acquired Flatfoot Deformity Confirmed via Magnetic Resonance Imaging: A Retrospective Study. J Foot Ankle Surg 2019;58(02):243-247 